\title{
Higher fatty liver index is associated with increased risk of new onset heart failure in healthy adults: $A$ nationwide population-based study in Korea
}

\author{
Jae-Hyung Roh
}

Chungnam National University School of Medicine Jae-Hyeong Park ( $\nabla$ jaehyeongpark@gmail.com )

Chungnam National University School of Medicine https://orcid.org/0000-0001-7035-286X

Hanbyul Lee

Kyungpook National University

\section{Yong-Hoon Yoon}

Chungnam National University School of Medicine

\section{Minsu Kim}

Chungnam National University School of Medicine

\section{Yong-Giun Kim}

Ulsan University Hospital

\section{Gyung-Min Park}

Ulsan University Hospital

Jae-Hwan Lee

Chungnam National University School of Medicine In-Whan Seong

Chungnam National University School of Medicine

\section{Research article}

Keywords: Fatty Liver Disease, Heart failure, Healthy people programs

Posted Date: November 20th, 2019

DOl: https://doi.org/10.21203/rs.2.17577/v1

License: (c) (1) This work is licensed under a Creative Commons Attribution 4.0 International License. Read Full License

Version of Record: A version of this preprint was published at BMC Cardiovascular Disorders on April 28th, 2020. See the published version at https://doi.org/10.1186/s12872-020-01444-x. 


\section{Abstract}

Background Heart failure (HF) is relatively common cardiovascular disease with high mortality and morbidity. Although it is associated with many cardiovascular risk factors, the association between nonalcoholic fatty liver disease (NAFLD), the most common chronic liver disease, and HF has not been evaluated in a large-scale cohort study. Thus, we evaluated the ability of the fatty liver Index (FLI), a surrogate marker of NAFLD, to predict the development of HF in healthy individuals.

Methods We evaluated 308,578 healthy persons without comorbidities who underwent the National Health check-ups in the republic of Korea from 2009 to 2014. The association between the FLI and HF was analyzed using multivariate Cox proportional-hazards models.

Results During a median of 5.4 years' follow-up, 2,532 subjects $(0.8 \%)$ were newly diagnosed with HF. Subjects were categorized into quartile groups according to FLI (Q1, 0-4.9; Q2, 5.0-12.5; Q3, 12.6-31.0; and Q4, >31.0). The cumulative incidence of HF was significantly higher in subjects with the highest FLI than in those with the lowest FLI (Q1, 307 [0.4\%] and Q4, 890 [1.2\%]; P<0.001). Adjusted hazard ratios (HRs) indicated that the highest FLI was independently associated with an increased risk for HF (HR between Q4 and Q1, 2.709; 95\% confidence interval=2.380-3.085; P<0.001). FLI was significantly associated with increased risk of new-onset HF regardless of their baseline characteristics.

Conclusions Higher FLI was independently associated with increased risk of HF in healthy Korean population.

\section{Introduction}

Non-alcoholic fatty liver disease (NAFLD) is the most common form of chronic liver disease. Its estimated prevalence is about $15-30 \%$ in general population [1]. The prevalence of NAFLD has been increasing along with the increase of obesity, insulin resistance and non-insulin dependent diabetes mellitus, and metabolic syndrome [2]. NAFLD can be found in about $60-70 \%$ among obese or diabetic population [1]. There are many evidences showing associations between NAFLD and established cardiovascular risk factors including abdominal obesity, dyslipidemia, insulin resistance and high blood pressure [3]. These cardiovascular risk factors are also components of metabolic syndrome. Thus, NAFLD is regarded as the hepatic manifestation of metabolic syndrome. Moreover, cardiovascular disease is the most common causes of death in patients with NAFLD, besides extrahepatic malignancies and liver-related complications $[4,5]$.

Heart failure (HF) is one of major cardiovascular diseases with higher morbidity, mortality, and healthcare costs around the world [6, 7]. The prevalence of $\mathrm{HF}$ is approximately $1-2 \%$ of the adult population in developed countries and its prevalence is rising among aged people $(\geq 10 \%$ among people $>70$ years of age) $[6,8]$. HF and NAFLD often coexist because they share the same risk factors and a similar pathophysiological processes $[9,10]$. HF may cause liver disease, and liver disease may leads to HF in the absence of other cardiovascular risk factors [10]. 
Although there are several small sized studies showing the co-existence of HF and NAFLD, they showed the simple association in several cross-sectional studies. We analyzed a large cohort consisting of a healthy Korean adult population without known traditional cardiovascular risk factors and co-morbidities to identify the association of NAFLD and new onset HF.

\section{Methods}

\section{Data sources}

We analyzed National Health Insurance Service-National Sample Cohort 2.0 (NHIS-NSC 2.0) data set. The Korean government has an obligatory public health insurance program, National Insurance Health Service (NIHS), including more than $97 \%$ of Korean people are affiliated. Total population ( $n=48,222,537)$ were classified into 2,142 classes according to their age, sex, area, eligibility status, and income level. Then, Korean government randomly selected $2.1 \%$ of them from each stratum $(n=1,021,208)$ from 2006 and made NHIS-NSC 2.0. It also included retrospective and prospective follow-up data which was collected from 2002 to 2015.[11] Because the NHIS covers about $97 \%$ of the total population of Korea, the NHISNSC 2.0 cohort is expected to represent the entire Korean population.

The cohort includes 4 datasets: the dataset of the sociodemographic information; the dataset of medical claims including information on the diagnosis based on the 10th revision of the International Classification of Disease (ICD-10) codes, admission, and treatment; the dataset of the National Health Screening of the cohort members; and the dataset of the medical institutions. The Korean government recommends the entire Korean adults to take the National Health check-up biennially including questionnaires on medical history and health-related behaviors including smoking status and alcohol consumption, chest X-ray, physical examinations and blood tests. About $72.1 \%$ of eligible population had National Health screening programs according to the 2013 NHIS statistics [11]. The cohort also includes mortality data from the death registration database of the Statistics Korea, a central government organization for statistics.

The NHIS-NSC 2.0 is open to any researchers if the NHIS review committee approves study protocol. This study was approved by the Institutional Review Board of the Chungnam National University Hospital, Daejeon, Korea (IRB No. 2019-10-053). Our IRB waived the requirement for informed consent.

\section{Study population}

We included population over 20 years of age having National Health check-ups at least one time from 2009 to 2014. We regarded the data from the first check-up as the index data, and the year of the index check-up as the index year. We excluded all subjects having pre-specified exclusion criteria; 1 ) those who diagnosed with cardiovascular diseases before the index year, 2) those who with valvular heart disease, 3) those who diagnosed with arrhythmias, HF, hypertension, diabetes, cerebrovascular disease, ischemic heart disease, hepatic disease, chronic pulmonary disease, chronic kidney disease, autoimmune disease, 
or peripheral artery disease, 4) those who with increased baseline systolic blood pressure (SBP) $140 \mathrm{mmHg}$ or diastolic blood pressure (DBP) $90 \mathrm{mmHg}$, and fasting blood glucose $126 \mathrm{mg} / \mathrm{dL}$ at the index check-up, and 5) those who with missing data in the index check-up.

\section{Definition of HF}

The primary outcome of this study was HF incidence according to FLI, and the incidence of HF was defined as the first occurrence during at least 2 different days of hospital visits, at HF admission, or death with a diagnosis of HF. We assessed each diagnosis based on the data from questionnaires, and the 1year claim data before the index year. When we used the claims data, we defined each diagnosis as the first occurrence during at least two different days of hospital visits (outpatient) or on the first admission, as likely a diagnosis of HF. The presence of HF was defined as those with "HF" according to the ICD-10 disease code in the claim dataset. HF patients who were assigned the following ICD-10 disease codes were considered as: "hypertensive heart disease with HF" (110.0), "hypertensive heart disease with hypertensive kidney disease with HF" (113.0),"hypertensive heart disease with hypertensive kidney disease with HF and kidney failure" (113.2), "ischemic cardiomyopathy" (I25.5), "dilated cardiomyopathy" (142.0), "cardiomyopathy, unspecified" (142.9), "cardiomyopathy in diseases classified elsewhere" (143), "HF" (I50) including "congestive HF" (I50.0), "left ventricular failure" (I50.1), and "HF, unspecified" (I50.9).

\section{Definition of data}

We calculated body mass index (BMI) with dividing weight $(\mathrm{kg})$ by height $(\mathrm{m})$-squared. Population with a BMI of $\geq 25 \mathrm{~kg} / \mathrm{m}^{2}$ wasregarded as obesity according to the World Health Organization guideline for the Asian population.[12] Smoking status was classified into 3 categories: non-smoker, ex-smoker, and current smoker. Alcohol consumption was evaluated with using standardized self-reporting questionnaires Data were censored at the time of HF occurrence, disqualification of the NHIS (death or immigration), or the end of the study (December 31th, 2015).

\section{Calculation fatty liver index}

We used a well validated, surrogate marker, FLI to identify patients with NAFLD [13]. FLI was calculated with 4 variables (triglycerides [TG], BMI, gamma-glutamyl transferase [GGT], and waist circumference $[W C])$ with following equation:

$F L I=\left(e^{0.953 \log _{e}(T G)+0.139 B M I+0.718 \log _{e}(G G T)+0.053 W C-15.745}\right) /\left(1+e^{0.953 \log } e^{(T G)+0.139 B M I+0.718 \log } e^{(G G T)+}\right.$ $0.053 W C-15.745) 100$

The original study showed that the FLI more than 60 as the cutoff for the diagnosis of fatty liver with positive likelihood ratio of 4.3 in general population.[13] Although the FLI is simple to calculate, and easy 
to screen fatty liver disease, there has been insufficient evidence regarding the diagnosis of fatty liver disease with FLI in Asians because of lower BMI and WC than other ethnic population [14]. Thus, we categorized our study group into quartiles according to their FLI and used quartile group in the statistical analysis.

\section{Statistical analysis}

We used continuous variables as mean \pm standard deviation and categorical parameters as number with percentage. We performed all statistical analysis with using $\mathrm{R}$ software version 3.3.3 (R Foundation for Statistical Computing, Vienna, Austria; www.r-project.org). Chi-square test and one-way analysis of variance test were used to evaluate statistical differences among the FLI quartiles. The calculation of cumulative event rates according to the FLI quartiles was done with Kaplan-Meier method and compared with a log-rank test. Adjusted hazard ratios (HR) and 95\% confidence interval (Cl) for HF incidence were estimated with Cox proportional hazard regression analysis. In the multivariate analysis, we adjusted age and sex in the model 1, and clinical characteristics associated with new onset HF of borderline statistical significance $(P<0.100)$ along with age and sex in the model 2. $P$ values of $<0.05$ were considered statistically significant.

\section{Results}

\section{Baseline characteristics of the participants}

We analyzed 308,578 subjects after the exclusion of the subjects who having the pre-specified exclusion criteria. The number of the subjects matching each exclusion criteria was presented in the Figure 1 . We divided our study population into 4 groups according to their FLI quartile values; first quartile (Q1), 0 - 4.9; second quartile (Q2), 5.0-12.5; third quartile (Q3), 12.6-31.0; and fourth quartile (Q4), >31.0. The comparison of baseline clinical characteristics and laboratory findings according to the FLI quartiles was summarized in the table 1. The subjects with higher FLI had a trend to higher age and higher incidence of male. BMI, waist circumference, blood pressures, the amount of alcohol consumption and the proportion of current smoker tended to increase from Q1 to Q4. Fasting glucose, total cholesterol, triglyceride and LDL cholesterol levels were increasing along with the increase of FLI quartiles.

\section{Association between FLI and the incidence of HF}

The total follow-up duration of this study cohort was median 5.4 years (interquartile range, 4.1 - 6.3), 2,532 subjects $(0.8 \%)$ had new onset HF. Table 2 shows the result of univariate analysis of new onset HF. Male gender, older age, higher BMI, higher total cholesterol, TG, and lower HDL cholesterol were associate significantly with new onset HF. Cumulative incidences of new onset HF according to the FLI quartiles were presented in the figure 2. The incidence of HF was significantly higher in the subjects with higher FLIs, compared to those with lower FLIs (Q1, 307 [0.4\%]; Q2, 543 [0.7\%]; Q3, 792 [1.0\%]; and Q4, 890 
[1.2\%], $\mathrm{P}<0.001$ by the Log-rank test). In multivariate models which were adjusted for age and sex in the model 1 and for clinical characteristics with borderline statistical significance along with age and sex in the model 2, the association between FLI quartile and HF incidence remained statistically significant in each model in the time-dependent Cox proportional hazard analsysis (Table 3 ). When we used various cutoff-points were tried to categorize the study population, which were suggested by the previous studies $(0 \leq \mathrm{FLI}<30,30 \leq \mathrm{FLI}<60$, and FLI $\geq 60$, by Bedogni et al. [13]; $0 \leq \mathrm{FLI}<25,25 \leq \mathrm{FLI}<35$, and FLI $\geq 35$ for male, $0 \leq \mathrm{FLI}<10,10 \leq \mathrm{FLI}<20$, and FLI $\geq 20$ for female, by Yang et al. [15]). Regardless of the cutoff-points used, the highest FLI group had the highest risk for new onset HF (Supplementary Table 1).

\section{Subgroup analysis}

Adjusted HRs according to subgroups summarized in the figure 3. FLI was a significant determinant of new onset HF in almost subgroups (Figure 3)..

\section{Discussion}

In this study, we showed higher FLI, a well validated index of NAFLD, was associated with increased risk of new onset HF in healthy Korean adult population. This association was found in most of the subgroups.

The association between fatty liver and HF has been observed in several studies [16, 17], and mildly elevated serum GGT levels in the absence of excessive alcohol consumption were long-term, independent predictors of incident HF in some large population-based studies [18-20]. Zhang et al. reported 37 had NAFLD defined by ultrasonography in 102 patients with HF with reduced ejection fraction (HFrEF) (36.3\%) [17]. In the Framingham study, higher GGT level, even within the normal range, had a 1.71-fold risk of HF $(95 \% \mathrm{Cl}=1.21-2.41)$ compared with participants with $\mathrm{GGT}$ concentrations less than the median. Also, GGT improved the risk reclassification modestly (net reclassification index, $5.7 \% ; P=0.01$ ) in the HF prediction [18]. In a prospective cohort study performed in Finland including 18,353 men and 19,726 women who were 25-74 years of age, moderate to high concentrations of serum GGT (from the 50th to the 90th percentiles) were significantly associated with incident $\mathrm{HF}$, and the predictive power was stronger in participants aged $<60$ years [20].

The presence of NAFLD was associated with higher BMI and left ventricular (LV) mass index, and had more severe LV fibrosis [17]. Other studies showed that patients with NAFLD had increased LV wall thickness and varying degrees of subclinical LV systolic or diastolic dysfunction [21, 22]. Increased LV wall thickness and fibrosis is a marker of LV diastolic dysfunction regardless of their symptoms. Jung et al. showed mild and moderate to severe NAFLD group had the higher risk of having abnormal LV relaxation compared with normal controls [21]. They showed increased odds ratios for abnormal LV relaxation [mild group: 1.29 (95\% confidence interval: 1.15 - 1.46), moderate to severe group: 1.95 (95\% confidence interval: 1.61 - 2.35)] and increased relative wall thickness (>0.42) [mild group: 1.26 (95\% confidence interval: 1.05 - 1.52), moderate to severe group: 1.46 (95\% confidence interval: $1.08-1.95)]$ in 
their cohort having 20,821 Korean adults received health check-ups. The proposed mechanism of increased LV wall thickness include insulin resistance [23], increased renin-angiotensin-aldosterone system (RAAS) [24], and endothelial dysfunction and inflammation [16]. Insulin resistance may contribute to the development of LV hypertrophy and HF through increased renal sodium retention and activation of the sympathetic nervous system $[25,26]$. Increased sympathetic nervous system can also increase hepatic fibrosis [27]. Mediators of RAAS, especially angiotensin II and aldosterone, play important roles in the development of hypertension and HF. Also, angiotensin II can be expressed by activated human hepatic stellate cells,[24] and activated local RAAS increase hepatic injury and induce fibrosis through angiotensin II-mediated stimulation of fibroblast proliferation and increased release of inflammatory cytokines [28]. Endothelial dysfunction, as a component of inflammation, can increase arterial stiffness and LV afterload through increased vascular tone, sympathetic overactivity, and sodium retention [29]. Inflammation can promote coronary atherosclerosis and increase risk for cardiomyopathy and conduction abnormalities [16]. Inflammatory mediators also affect the progression of fatty liver disease via impaired fatty acid oxidation, increased oxidative stress, and local inflammation [30].

The presence of NAFLD was associated with poor prognosis of chronic HF [31] and acute HF [32]. Although mechanisms of the association between NAFLD and poor clinical outcomes of HF remains unclear, there are several proposed explanations including increased coronary atherosclerosis, enhancing LV hypertrophy and dysfunction, and increased aortic valve calcification [33-35]. Because liver plays an important role in the regulation of antioxidant and anti-inflammation systems [36], hepatic dysfunction can increase chronic inflammation and oxidative tissue injury in patient with HF [37] and the impaired antioxidant and antiinflammation roles of liver may further deteriorate the prognosis of HF patients [38].

\section{Limitations}

Our study has several limitations. First, we estimated new onset HF by using the NHIS-NSC 2.0 database, which is based on ICD-10 disease code, and the accuracy of HF diagnosis was not validated in this study. Further studies should be needed to validate HF patients with health insurance data using either hospital-based medical records. Second, we can not establish a causal relationship between increased FLI and increased HF incidence because this study is an observational study. Finally, this study population included only Koreans. Thus, caution should be needed in the generalization of this data to other ethnic populations.

\section{Conclusions}

Our findings demonstrated that higher FLI was independently associated with increased risk for HF in healthy Korean population.

\section{Clinical Perspectives}


Because control of metabolic syndrome and prevention of HF are important in the reduction of cardiovascular morbidity and mortality, the identification of increased HF risk may have important public health implications. Further studies are needed to document the effect of management of NAFLD not only on the risk of new onset HF, but also on the reduction of cardiovascular morbidity and mortality.

\section{Declarations}

\section{Ethics approval and consent to participate}

Institutional Review Board of Chungnam National University Hosptial approved this study protocol (IRB No. 2019-10-053). Our IRB waived the requirement for informed consent.

\section{CONSENT FOR PUBLICATION}

Not applicable

\section{AVAILABILITY OF DATA AND MATERIAL}

The datasets used and analyzed during the present study are available from the National Health Insurance Service. It can be used after acquisition of the right to use this data.

\section{COMPETING INTERESTS}

None. The authors have nothing to declare.

\section{FUNDING}

This work was supported by research fund of Chungnam National University

\section{AUTHOR CONTRIBUTIONS}

RJH and PJH: study conception and design, acquisition of data, interpretation of data, drafting of manuscript, and critical revision; LHB: acquisition of data, analysis and interpretation of data; and YYH, KMS, KYG, PGM, LJH, and SIW: interpretation of data, and critical revision.

\section{References}


1.Targher G, Day CP, Bonora E. Risk of cardiovascular disease in patients with nonalcoholic fatty liver disease. N Engl J Med. 2010;363:1341-50.

2.McPherson S, Hardy T, Henderson E, Burt AD, Day CP, Anstee QM. Evidence of NAFLD progression from steatosis to fibrosing-steatohepatitis using paired biopsies: implications for prognosis and clinical management. J Hepatol. 2015;62:1148-55.

3.Angulo P. Nonalcoholic fatty liver disease. N Engl J Med. 2002;346:1221-31.

4.Ekstedt $M$, Hagstrom $H$, Nasr P, Fredrikson M, Stal P, Kechagias $S$, et al. Fibrosis stage is the strongest predictor for disease-specific mortality in NAFLD after up to 33 years of follow-up. Hepatology. 2015;61:1547-54.

5.Angulo P, Kleiner DE, Dam-Larsen S, Adams LA, Bjornsson ES, Charatcharoenwitthaya P, et al. Liver Fibrosis, but No Other Histologic Features, Is Associated With Long-term Outcomes of Patients With Nonalcoholic Fatty Liver Disease. Gastroenterology. 2015;149:389-97 e10.

6.Ponikowski P, Voors AA, Anker SD, Bueno H, Cleland JGF, Coats AJS, et al. 2016 ESC Guidelines for the diagnosis and treatment of acute and chronic heart failure: The Task Force for the diagnosis and treatment of acute and chronic heart failure of the European Society of Cardiology (ESC)Developed with the special contribution of the Heart Failure Association (HFA) of the ESC. Eur Heart J. 2016;37:21292200.

7.Lee JH, Kim MS, Kim EJ, Park DG, Cho HJ, Yoo BS, et al. KSHF Guidelines for the Management of Acute Heart Failure: Part I. Definition, Epidemiology and Diagnosis of Acute Heart Failure. Korean Circ J. 2019;49:1-21.

8.Lee JH, Lim NK, Cho MC, Park HY. Epidemiology of Heart Failure in Korea: Present and Future. Korean Circ J. 2016;46:658-664.

9.Moller S, Bernardi M. Interactions of the heart and the liver. Eur Heart J. 2013;34:2804-11.

10.Xanthopoulos A, Starling RC, Kitai T, Triposkiadis F. Heart Failure and Liver Disease: Cardiohepatic Interactions. JACC Heart Fail. 2019;7:87-97.

11.Lee J, Lee JS, Park SH, Shin SA, Kim K. Cohort Profile: The National Health Insurance Service-National Sample Cohort (NHIS-NSC), South Korea. Int J Epidemiol. 2017;46:e15.

12.Consultation WHOE. Appropriate body-mass index for Asian populations and its implications for policy and intervention strategies. Lancet. 2004;363:157-63.

13.Bedogni G, Bellentani S, Miglioli L, Masutti F, Passalacqua M, Castiglione A, et al. The Fatty Liver Index: a simple and accurate predictor of hepatic steatosis in the general population. BMC Gastroenterol. 2006;6:33. 
14.Grundy SM, Cleeman JI, Daniels SR, Donato KA, Eckel RH, Franklin BA, et al. Diagnosis and management of the metabolic syndrome: an American Heart Association/National Heart, Lung, and Blood Institute Scientific Statement. Circulation. 2005;112:2735-52.

15.Yang BL, Wu WC, Fang KC, Wang YC, Huo TI, Huang YH, et al. External validation of fatty liver index for identifying ultrasonographic fatty liver in a large-scale cross-sectional study in Taiwan. PLoS One. 2015;10:e0120443.

16.Anstee QM, Mantovani A, Tilg H, Targher G. Risk of cardiomyopathy and cardiac arrhythmias in patients with nonalcoholic fatty liver disease. Nat Rev Gastroenterol Hepatol. 2018;15:425-439.

17.Zhang Z, Wang P, Guo F, Liu X, Luo T, Guan Y, et al. Chronic heart failure in patients with nonalcoholic fatty liver disease: prevalence, clinical features, and relevance. J Int Med Res. 2018;46:3959-3969.

18.Dhingra R, Gona P, Wang TJ, Fox CS, D’Agostino RB, Sr., Vasan RS. Serum gamma-glutamyl transferase and risk of heart failure in the community. Arterioscler Thromb Vasc Biol. 2010;30:1855-60.

19.Wannamethee SG, Whincup PH, Shaper AG, Lennon L, Sattar N. Gamma-glutamyltransferase, hepatic enzymes, and risk of incident heart failure in older men. Arterioscler Thromb Vasc Biol. 2012;32:830-5.

20.Wang Y, Tuomilehto J, Jousilahti P, Salomaa V, Li B, Antikainen R, et al. Serum gammaglutamyltransferase and the risk of heart failure in men and women in Finland. Heart. 2013;99:163-7.

21.Jung JY, Park SK, Ryoo JH, Oh CM, Kang JG, Lee JH, et al. Effect of non-alcoholic fatty liver disease on left ventricular diastolic function and geometry in the Korean general population. Hepatol Res. 2017:47:522-532.

22.Trovato FM, Martines GF, Catalano D, Musumeci G, Pirri C, Trovato GM. Echocardiography and NAFLD (non-alcoholic fatty liver disease). Int J Cardiol. 2016;221:275-9.

23.Saad MF, Rewers M, Selby J, Howard G, Jinagouda S, Fahmi S, et al. Insulin resistance and hypertension: the Insulin Resistance Atherosclerosis study. Hypertension. 2004;43:1324-31.

24.Bataller R, Sancho-Bru P, Gines P, Lora JM, Al-Garawi A, Sole M, et al. Activated human hepatic stellate cells express the renin-angiotensin system and synthesize angiotensin II. Gastroenterology. 2003;125:117-25.

25.Reaven GM, Hoffman BB. A role for insulin in the aetiology and course of hypertension? Lancet. $1987 ; 2: 435-7$.

26.Soleimani M. Insulin resistance and hypertension: new insights. Kidney Int. 2015;87:497-9.

27.Sigala B, McKee C, Soeda J, Pazienza V, Morgan M, Lin Cl, et al. Sympathetic nervous system catecholamines and neuropeptide $Y$ neurotransmitters are upregulated in human NAFLD and modulate 
the fibrogenic function of hepatic stellate cells. PLoS One. 2013;8:e72928.

28.Warner FJ, Lubel JS, McCaughan GW, Angus PW. Liver fibrosis: a balance of ACEs? Clin Sci (Lond). 2007;113:109-18.

29.Brandes RP. Endothelial dysfunction and hypertension. Hypertension. 2014;64:924-8.

30.Browning JD and Horton JD. Molecular mediators of hepatic steatosis and liver injury. J Clin Invest. 2004;114:147-52.

31.Takahashi T, Watanabe T, Shishido T, Watanabe K, Sugai T, Toshima T, et al. The impact of nonalcoholic fatty liver disease fibrosis score on cardiac prognosis in patients with chronic heart failure. Heart Vessels. 2018;33:733-739.

32.Valbusa F, Agnoletti D, Scala L, Grillo C, Arduini P, Bonapace S, et al. Non-alcoholic fatty liver disease and increased risk of all-cause mortality in elderly patients admitted for acute heart failure. Int $\mathrm{J}$ Cardiol. 2018;265:162-168.

33.Targher G, Byrne CD, Lonardo A, Zoppini G, Barbui C. Non-alcoholic fatty liver disease and risk of incident cardiovascular disease: A meta-analysis. J Hepatol. 2016;65:589-600.

34.VanWagner LB, Wilcox JE, Colangelo LA, Lloyd-Jones DM, Carr JJ, Lima JA, et al. Association of nonalcoholic fatty liver disease with subclinical myocardial remodeling and dysfunction: A populationbased study. Hepatology. 2015;62:773-83.

35.Mantovani A, Pernigo M, Bergamini C, Bonapace S, Lipari P, Valbusa F, et al. Heart valve calcification in patients with type 2 diabetes and nonalcoholic fatty liver disease. Metabolism. 2015;64:879-87.

36.Valentova M, von Haehling S, Doehner W, Murin J, Anker SD, Sandek A. Liver dysfunction and its nutritional implications in heart failure. Nutrition. 2013;29:370-8.

37.Sawyer DB. Oxidative stress in heart failure: what are we missing? Am J Med Sci. 2011;342:120-4.

38.Niebauer J, Volk HD, Kemp M, Dominguez M, Schumann RR, Rauchhaus M, et al. Endotoxin and immune activation in chronic heart failure: a prospective cohort study. Lancet. 1999;353:1838-42.

\section{Tables}

Table 1. Baseline characteristics and laboratory findings 


\section{$\begin{array}{llll}\text { Q1 } & \text { Q2 } & \text { Q3 } & \text { Q4 }\end{array}$}

\section{Baseline characteristics}

Age, years

$36.7 \pm 11.4$

$41.9 \pm 12.4$

$44.0 \pm 12.4$

$43.1 \pm 11.3<0.001$

$<0.001$

Male sex (\%)

$12,102(15.7) \quad 30,737(39.8) \quad 46,692(60.5)$

$62,507(81.0)<0.001$

$<0.001$

Height, cm

$161.4 \pm 7.2$

Weight, $\mathrm{Kg}$

$52.2 \pm 6.1$

$163.4 \pm 8.9$

$165.6 \pm 9.3$

$168.8 \pm 8.4<0.001$

$<0.001$

BMI, $\mathrm{Kg} / \mathrm{m}^{2}$

$20.0 \pm 1.7$

$59.1 \pm 7.3$

$65.4 \pm 8.3$

$75.0 \pm 10.4$

$<0.001$

$<0.001$

Waist circumference, cm

$67.9 \pm 4.9$

$22.1 \pm 1.8$

$23.8 \pm 2.1$

$26.3 \pm 2.8$

$<0.001$

$<0.001$

WHR, \%

$0.3 \pm 0.0$

$74.9 \pm 4.8$

$80.5 \pm 5.1$

$87.6 \pm 6.5$

$<0.001$

$<0.001$

$\mathrm{SBP}, \mathrm{mmHg}$

111.0 \pm 11.1

$0.4 \pm 0.0$

$0.4 \pm 0.0$

$0.4 \pm 0.1$

$<0.001$

$<0.001$

DBP , mmHg

$69.4 \pm 8.0$

$115.4 \pm 11.1$

$118.6 \pm 10.6$

$121.8 \pm 10.0$

$<0.001$

$<0.001$

Smoking

$$
72.0 \pm 7.9
$$

$74.0 \pm 7.6$

$76.3+7.1$

$<0.001$

$<0.001$

$<0.001$

$<0.001$

Non-smoker

$65,184(84.5) \quad 53,779(69.7) \quad 42,729(55.4) \quad 28,355(36.8)$

Ex-smoker

$3,609(4.7)$

$6,720(8.7)$

$10,711(13.9)$

14,058(18.2)

Current-smoker

$8,350(10.8)$

$16,638(21.6)$

$23,713(30.7)$

$34,732(45.0)$

Alcohol consumption

Amount, g/week

$31.6 \pm 77.4$

$49.5 \pm 102.2$

$73.9 \pm 129.6$

$122.5 \pm 174.0<0.001$

$<0.001$

Activity, met-min/week

$346.7 \pm 350.8$

$379.7 \pm 382.7$

$388.8 \pm 388.1$

$374.6 \pm 373.4<0.001$

$<0.001$

\section{Laboratory findings}

AST, IU/L

$19.8 \pm 8.0$

$21.7 \pm 12.4$

$23.7 \pm 16.7$

$29.4 \pm 33.0$

$<0.001$

$<0.001$

$\mathrm{ALT}, \mathrm{IU} / \mathrm{L}$

$14.5 \pm 8.9$

$17.9 \pm 12.2$

$22.9 \pm 22.2$

$36.3 \pm 50.5$

$<0.001$

$<0.001$

GGT, IU/L

$14.2 \pm 5.6$

$19.1 \pm 9.9$

$28.1 \pm 20.3$

$60.3 \pm 62.4$

$<0.001$

$<0.001$

Hemoglobin, g/dL

$13.0 \pm 1.4$

$13.6 \pm 1.6$

$14.2 \pm 1.6$

$14.9 \pm 1.4$

$<0.001$

$<0.001$

Fasting glucose, mg/dL

$87.9 \pm 9.5$

$90.3 \pm 10.2$

$92.2 \pm 10.7$

$94.8 \pm 11.4$

$<0.001$

$<0.001$

Total cholesterol, mg/dL

$176.9 \pm 33.6$

$187.1 \pm 35.9$

$196.2 \pm 39.3$

$207.0 \pm 40.7$

$<0.001$

$<0.001$

Triglyceride, mg/dL

$62.6 \pm 22.5$

$88.0 \pm 34.0$

$119.9 \pm 53.6$

$197.6 \pm 132.6$

$<0.001$

$<0.001$

HDL cholesterol, mg/dL

$64.1 \pm 24.8$

$59.3 \pm 19.9$

$55.5 \pm 25.8$

$51.7 \pm 32.0$

$<0.001$

$<0.001$

LDL cholesterol, mg/dL

$109.4 \pm 257.2$

$113.6 \pm 143.5$

$121.2 \pm 134.2$

$122.6 \pm 129.0$

$<0.001$

$<0.001$

Creatinine, $\mathrm{mg} / \mathrm{dL}$

$0.9 \pm 1.0$

$1.0 \pm 1.1$

$1.0 \pm 1.1$

$1.1 \pm 1.2$

$<0.001$

$<0.001$ 
ALT: alanine aminotransferase, AST: aspartate aminotransferase, BMI: body mass index, DBP: diastolic blood pressure, GFR: glomerular filtration rate, GGT: gamma-glutamyl transferase, HDL: high-density lipoprotein, LDL: low-density lipoprotein, SBP: systolic blood pressure, WHR: waist-hip ratio

Table 2. Univariate analysis of new onset heart failure 


\begin{tabular}{|c|c|c|c|c|c|c|c|}
\hline \multirow[t]{2}{*}{ Characteristics } & \multirow[t]{2}{*}{ Value } & \multirow[t]{2}{*}{$\mathrm{N}$} & \multirow[t]{2}{*}{ HF (\%) } & \multirow[t]{2}{*}{$\mathrm{HR}$} & \multicolumn{2}{|c|}{$95 \% \mathrm{CI}$} & \multirow[t]{2}{*}{ P-value } \\
\hline & & & & & Lower & Upper & \\
\hline Total & Total & 308,578 & $2,532(0.8)$ & & & & \\
\hline \multirow[t]{2}{*}{ Sex } & Female & 156,540 & $1,263(0.8)$ & \multicolumn{4}{|c|}{ Reference } \\
\hline & Male & 152,038 & $1,269(0.8)$ & 1.113 & 1.030 & 1.204 & 0.007 \\
\hline \multirow[t]{6}{*}{ Age, year } & $20-34$ & 99,774 & $119(0.1)$ & \multicolumn{4}{|c|}{ Reference } \\
\hline & $35-49$ & 130,099 & $637(0.5)$ & 3.908 & 3.213 & 4.753 & $<0.001$ \\
\hline & $50-64$ & 65,907 & $1,067(1.6)$ & 12.536 & 10.371 & 15.153 & $<0.001$ \\
\hline & $65-74$ & 10,374 & $497(4.8)$ & 35.791 & 29.298 & 43.722 & $<0.001$ \\
\hline & $\geq 75$ & 2,424 & $212(8.7)$ & 75.652 & 60.429 & 94.711 & $<0.001$ \\
\hline & Continuous & & & 1.087 & 1.084 & 1.090 & $<0.001$ \\
\hline \multirow[t]{6}{*}{ BMI, $\mathrm{Kg} / \mathrm{m} 2$} & $<18.5$ & 16,662 & $101(0.6)$ & 1.075 & 0.870 & 1.328 & 0.505 \\
\hline & 18.5-19.9 & 33,181 & $177(0.5)$ & 0.929 & 0.786 & 1.098 & 0.390 \\
\hline & $20-22.4$ & 89,515 & $625(0.7)$ & \multicolumn{4}{|c|}{ Reference } \\
\hline & $22.5-24.9$ & 90,961 & $814(0.9)$ & 1.115 & 1.005 & 1.238 & 0.041 \\
\hline & $\geq 25$ & 78,259 & $815(1.0)$ & 1.417 & 1.276 & 1.573 & $<0.001$ \\
\hline & Continuous & & & 1.058 & 1.045 & 1.072 & $<0.001$ \\
\hline \multirow[t]{3}{*}{ Smoking } & Non-smoker & 190,047 & $1,599(0.8)$ & \multicolumn{4}{|c|}{ Reference } \\
\hline & Ex-smoker & 35,098 & $320(0.9)$ & 0.934 & 0.812 & 1.074 & 0.340 \\
\hline & Current-smoker & 83,433 & $613(0.7)$ & 1.120 & 0.997 & 1.259 & 0.057 \\
\hline \multirow[t]{4}{*}{ Alcohol consumption } & 0 & 149,779 & $1,557(1.0)$ & \multicolumn{4}{|c|}{ Reference } \\
\hline & $<=140$ & 110,537 & $626(0.6)$ & 0.882 & 0.798 & 0.975 & 0.014 \\
\hline & $>140$ & 48,262 & $349(0.7)$ & 0.991 & 0.871 & 1.126 & 0.890 \\
\hline & Continuous & & & 1.000 & 1.000 & 1.000 & 0.960 \\
\hline \multirow[t]{3}{*}{$\mathrm{SBP}, \mathrm{mmHg}$} & $<120$ & 165,193 & $1,019(0.6)$ & \multicolumn{4}{|c|}{ Reference } \\
\hline & $120-139$ & 143,385 & $1,513(1.1)$ & 1.295 & 1.195 & 1.404 & $<0.001$ \\
\hline & Continuous & & & 1.013 & 1.010 & 1.017 & $<0.001$ \\
\hline \multirow[t]{3}{*}{$\mathrm{DBP}, \mathrm{mmHg}$} & $<80$ & 210,638 & $1,513(0.7)$ & \multicolumn{4}{|c|}{ Reference } \\
\hline & $80-89$ & 97,940 & $1,019(1.0)$ & 1.220 & 1.126 & 1.322 & $<0.001$ \\
\hline & Continuous & & & 1.016 & 1.011 & 1.022 & $<0.001$ \\
\hline \multirow[t]{3}{*}{$\mathrm{FBS}, \mathrm{mg} / \mathrm{dL}$} & $<100$ & 246,415 & $1,876(0.8)$ & \multicolumn{4}{|c|}{ Reference } \\
\hline & $100-125$ & 62,163 & $656(1.1)$ & 1.042 & 0.953 & 1.140 & 0.370 \\
\hline & Continuous & & & 1.003 & 1.000 & 1.007 & 0.058 \\
\hline \multirow[t]{3}{*}{ Activity, met-min/week } & $<500$ & 221107 & $1871(0.8)$ & & & & \\
\hline & 500-999 & 65361 & $453(0.7)$ & 0.877 & 0.791 & 0.972 & 0.013 \\
\hline & $\geq 1000$ & 22110 & $208(0.9)$ & 0.890 & 0.770 & 1.027 & 0.11 \\
\hline Waist circumference, $\mathrm{cm}$ & Continuous & & & 1.027 & 1.022 & 1.032 & $<0.001$ \\
\hline
\end{tabular}




\begin{tabular}{llrrrc} 
Total cholesterol, mg/dL & Continuous & 1.001 & 1.000 & 1.002 & $<0.001$ \\
Triglyceride, mg/dL & Continuous & 1.001 & 1.000 & 1.001 & $<0.001$ \\
HDL-cholesterol, mg/dL & Continuous & 0.997 & 0.995 & 0.999 & 0.014 \\
LDL-cholesterol, mg/dL & Continuous & 1.000 & 1.000 & 1.000 & 0.29 \\
\hline
\end{tabular}

BMI: body mass index, DBP: diastolic blood pressure, FBS: fasting blood glucose, HDL: high-density lipoprotein, HF: heart failure, LDL: low-density lipoprotein, SBP: systolic blood pressure

Table 3. Association between fatty liver index and new onset heart failure

\begin{tabular}{|c|c|c|c|c|c|c|c|c|c|c|c|}
\hline \multirow[t]{2}{*}{ FLI } & \multirow[t]{2}{*}{ N. } & \multirow{2}{*}{$\begin{array}{c}\text { New } \\
\text { onset HF }\end{array}$} & \multicolumn{3}{|c|}{ Univariate } & \multicolumn{3}{|c|}{ Model 1* } & \multicolumn{3}{|c|}{ Model $2^{\dagger}$} \\
\hline & & & $\mathrm{HR}$ & $95 \%$ CI & $\begin{array}{c}\mathrm{P}- \\
\text { value }\end{array}$ & $\mathrm{HR}$ & 95\% CI & $\begin{array}{c}\text { P- } \\
\text { value }\end{array}$ & $\mathrm{HR}$ & $\begin{array}{c}95 \% \\
\mathrm{CI}\end{array}$ & $\begin{array}{c}\mathrm{P}- \\
\text { value }\end{array}$ \\
\hline $\begin{array}{l}\text { FLI } \\
\text { quartile }\end{array}$ & & & & & & & & & & & \\
\hline $\begin{array}{l}\mathrm{Q} 1(0- \\
4.9)\end{array}$ & 77,143 & $307(0.4)$ & & Reference & & & Reference & & & Referenc & \\
\hline $\begin{array}{l}\text { Q2 (5.0 - } \\
12.5)\end{array}$ & 77,137 & $543(0.7)$ & 1.691 & $\begin{array}{l}1.470- \\
1.945\end{array}$ & $<0.001$ & 1.104 & $\begin{array}{l}0.958- \\
1.271\end{array}$ & 0.171 & 1.071 & $\begin{array}{l}0.930- \\
1.234\end{array}$ & 0.339 \\
\hline $\begin{array}{l}\text { Q3 (12.6 - } \\
31.0)\end{array}$ & 77,153 & $792(1.0)$ & 2.409 & $\begin{array}{l}2.112- \\
2.748\end{array}$ & $<0.001$ & 1.386 & $\begin{array}{l}1.212- \\
1.586\end{array}$ & $<0.001$ & 1.314 & $\begin{array}{l}1.147- \\
1.504\end{array}$ & $<0.001$ \\
\hline $\begin{array}{l}\text { Q4 (> } \\
31.0)\end{array}$ & 77,145 & $890(1.2)$ & 2.709 & $\begin{array}{l}2.380- \\
3.085\end{array}$ & $<0.001$ & 1.857 & $\begin{array}{l}1.620- \\
2.127\end{array}$ & $<0.001$ & 1.710 & $\begin{array}{l}1.489- \\
1.964\end{array}$ & $<0.001$ \\
\hline
\end{tabular}

${ }^{*}$ Cox proportional hazard models including age, and sex as covariates

${ }^{\dagger}$ Cox proportional hazard models including age, sex, and clinical characteristics, of which association with new onset heart failure showed borderline statistical significance $(\mathrm{P}<0.100)$

CI: confidential interval, FLI: fatty liver index, HR: hazard ratio, REF: reference

\section{Figures}


556,884 participants who underwent the National Health checkups from Jan. 2009 to Dec. 2014

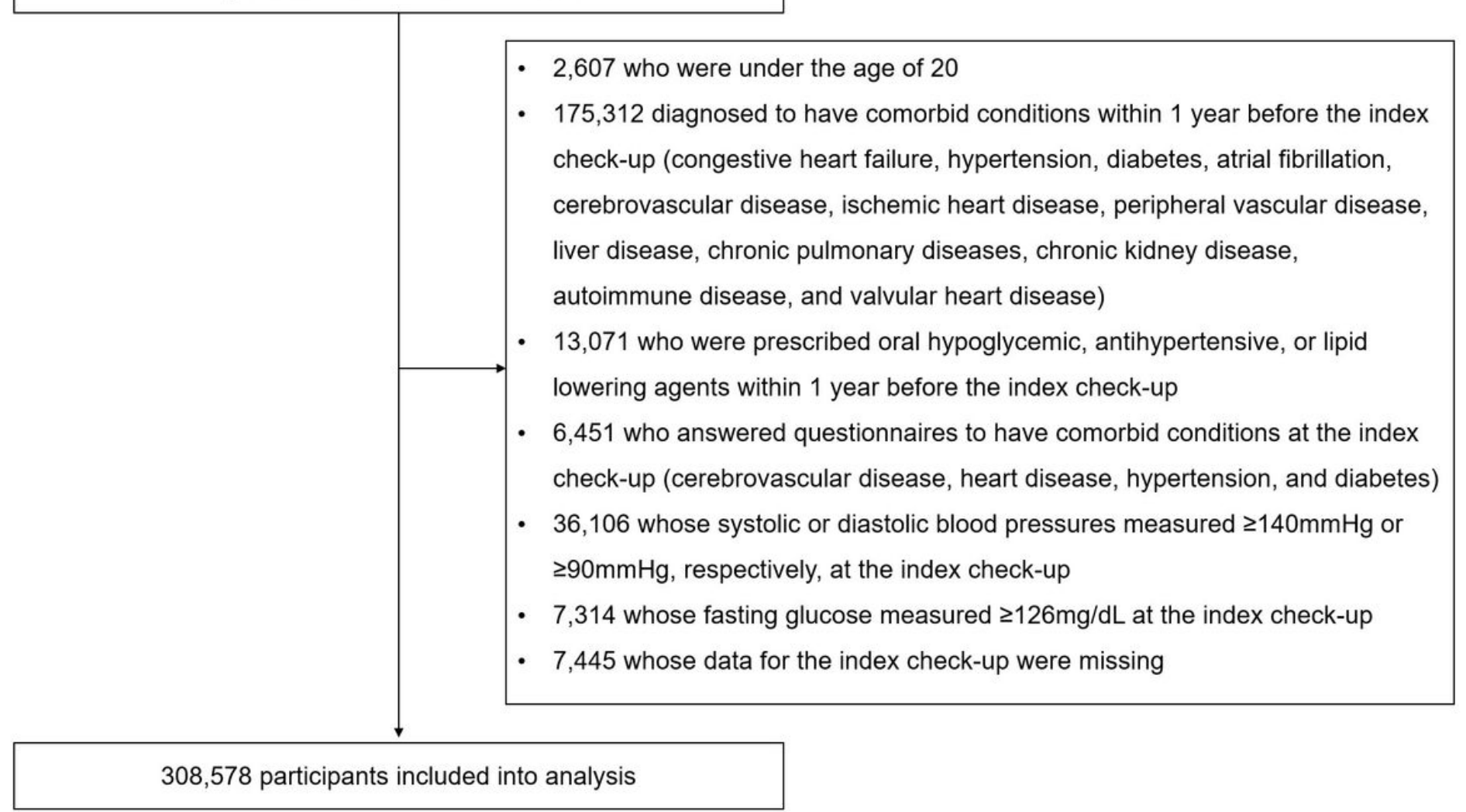

\section{Figure 1}

Overview of the study population 


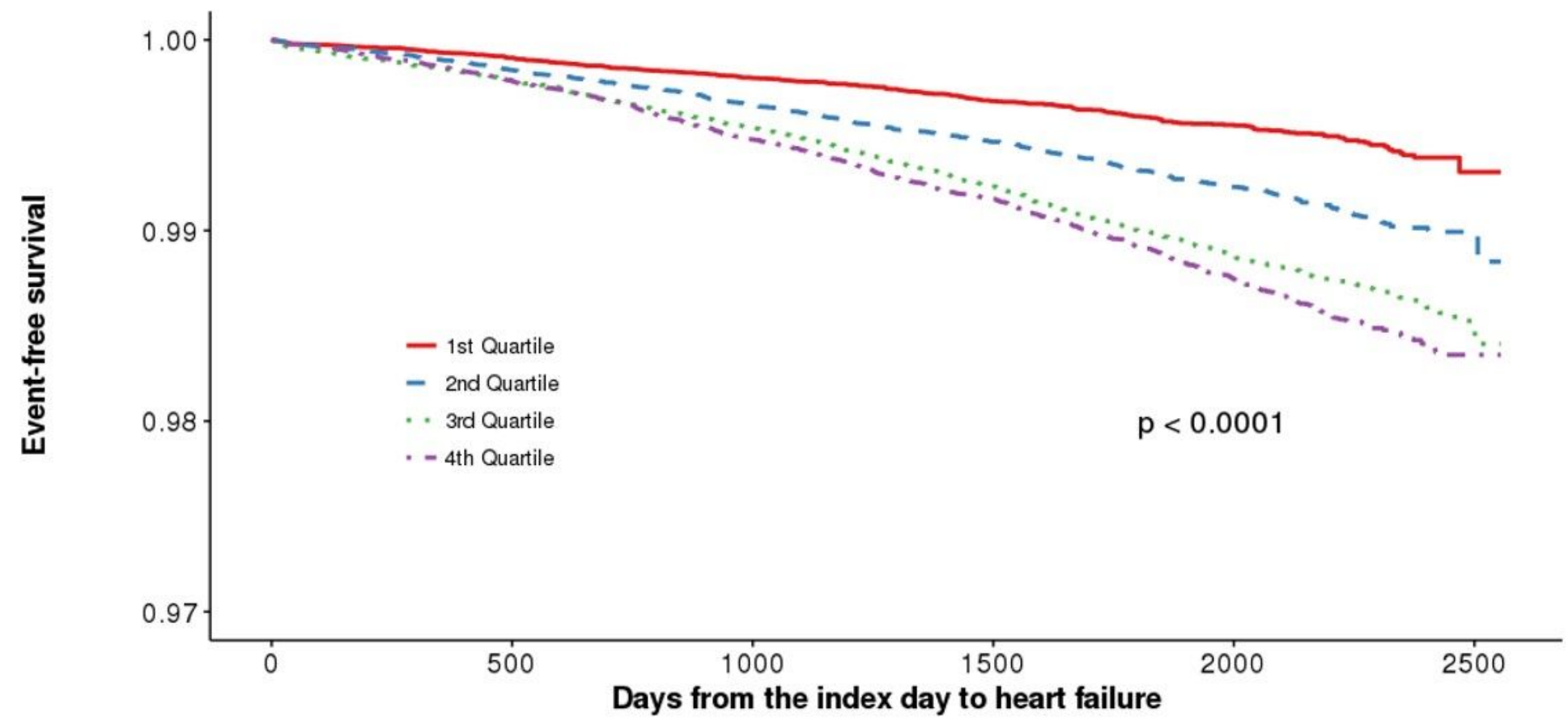

Number at risk

\begin{tabular}{|c|c|c|c|c|c|}
\hline $\begin{array}{l}\text { 1st Quartile- } 77143 \\
\text { 2nd Quartile- } 77137 \\
\text { 3rd Quartile- } 77153 \\
\text { 4th Quartile- } 77145\end{array}$ & $\begin{array}{l}73291 \\
74041 \\
74404 \\
74148\end{array}$ & $\begin{array}{l}64459 \\
66734 \\
67701 \\
67523\end{array}$ & $\begin{array}{l}52624 \\
55826 \\
57300 \\
57230\end{array}$ & $\begin{array}{l}33140 \\
36734 \\
38947 \\
38927\end{array}$ & $\begin{array}{l}331 \\
636 \\
820 \\
701\end{array}$ \\
\hline
\end{tabular}

Figure 2

Cumulative incidence of new onset heart failure according to their quartiles. Statistical significance was determined using the log-rank test. 


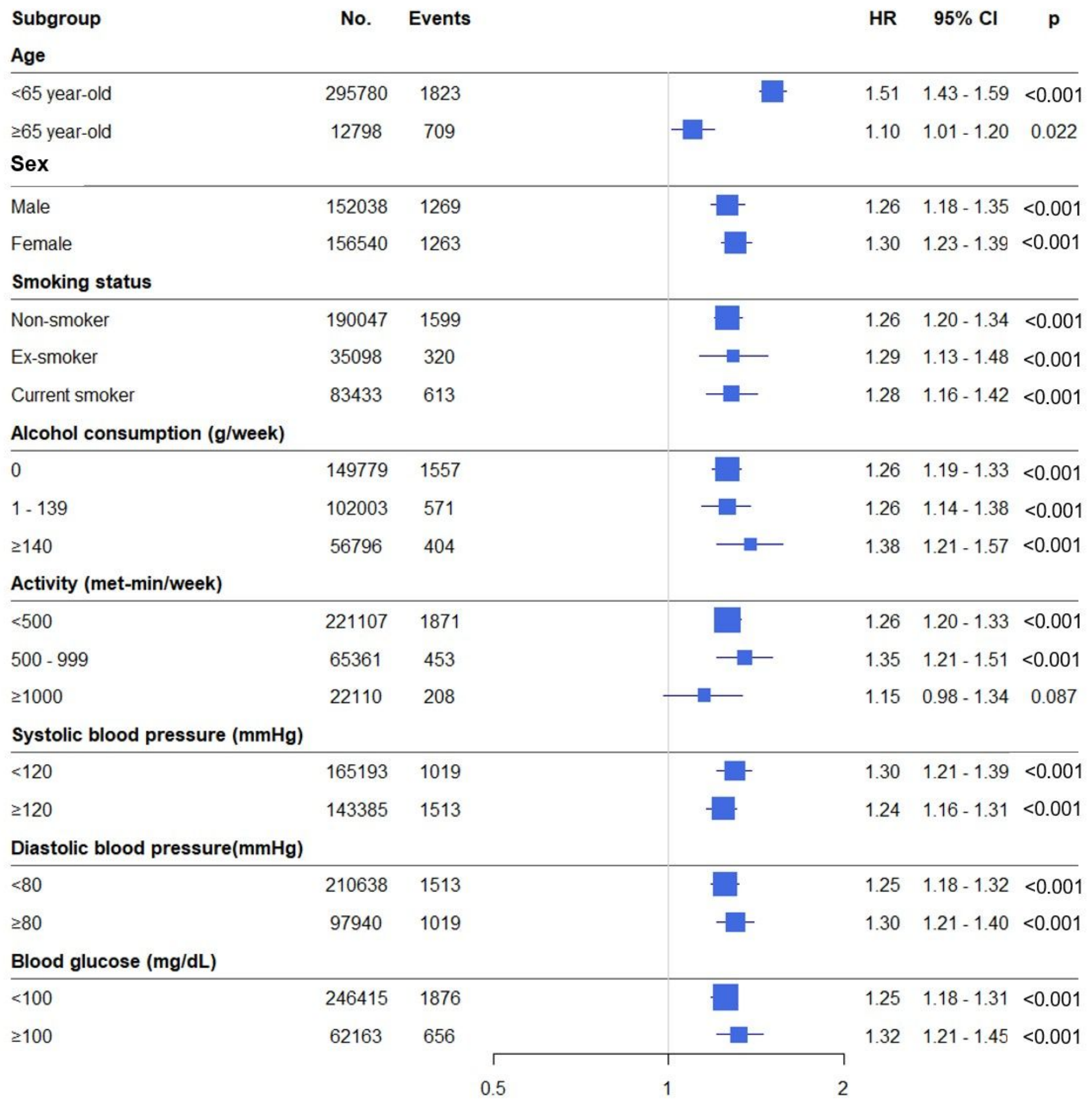

\section{Figure 3}

Forest plots of hazard ratios for new onset heart failure stratified by various clinical characteristics. $\mathrm{Cl}=$ confidence interval; $\mathrm{HR}=$ hazard ratio.

\section{Supplementary Files}

This is a list of supplementary files associated with this preprint. Click to download. 
- SupTable1.docx

Page 19/19 